\title{
Exploration and Practice of Innovative Education in the Comprehensive Experiment of Inorganic Materials
}

\author{
Ya-li WANG*, Su-ping CUI, Ying-liang TIAN, Jian-hua YAN and Xiao-yu MA \\ College of Materials Science and Engineering, Beijing University of Technology, Beijing, CHINA \\ ${ }^{*}$ Corresponding author
}

Keywords: Inorganic material specialty, Cultivation of innovative talents, Experimental teaching, Teaching model.

\begin{abstract}
In our university, 'The comprehensive experiment of inorganic materials' is professional courses of inorganic material specialty. It trains the ability and quality of students to solve the problems of engineering and scientific research. By improving the teaching content, the teaching plan, evaluation model and evaluation methods, it formed complete innovation practice teaching mode. The teaching mode consist of 'thick foundation interest, emphasizing the comprehensive, intensifying experiment, autonomous learning, improve ability, continuous innovation', it promote and stimulate students' innovation ability.
\end{abstract}

\section{《无机材料综合实验》的创新教育探索与实践}

王亚丽 ${ }^{\star}$, 崔素萍, 田英良, 严建华, 马晓宇

北京工业大学材料科学与工程学院, 朝阳区, 北京, 中国

“通讯作者

关键词: 无机材料专业; 创新人才培养; 实验教学; 教学模式

摘要：我校无机材料专业课程《无机材料综合实验》，培养学生解决工程及科学研究问题的 能力与素质。通过改进现有的教学内容、教学方案、评价模式和考核方式, 形成了“厚实基础、 注重兴趣、强调综合、强化实验、自主学习、提高能力、不断创新”的完整创新实践教学模式, 促进和激发学生的创新能力。

\section{1. 引言}

无机材料专业卓越工程师是要培养具有较强的工程实践能力、自我获取知识能力、社会 交往能力、组织管理能力和国际化视野的无机材料工程型人才。在掌握材料科学与工程领域 所需要的基础知识的基础上, 使学生树立材料与环境协调发展的理念, 具备扎实的自然科学 基础、人文社会科学基础和材料科学与工程专业基础知识; 掌握材料科学与工程的基本理论、 研究方法和生产工艺技术, 具备较强的无机材料技术开发、技术咨询、技术服务、工程设计 与工程实施能力; 能在无机材料科技型企业（研究、设计院）、生产型企业、咨询服务型企 业等, 从事新产品研究、技术开发、工艺设计、技术改造、工程应用等方面的工作。按照学 校统一部署, 为了做好工程专业认证准备和适应材料科学与工程专业实验班培养的要求, 依 据我校“立足北京，融入北京，辐射全国、面向世界”的定位目标，结合北京市经济发展需求 和材料产业结构调整, 制定了《北京工业大学材料科学与工程专业2015版培养方案》。其中 对原来的 1 个学分的专业实验课《无机材料实验》进行修订，更改为 3 个学分的专业综合实验 
课《无机材料综合实验》, 相应学时由原来的 24 学时提高到48学时。这门课要求学生在完成 无机材料学科的基础课和专业基础课后选修, 让学生加强对无机材料的制备、性能检测方法 的认识, 熟悉基本实验设备的使用, 并能亲手实验, 增强对材料的感性认识。课程修订之前, 我们的实验指导教师面对很少的实验课时, 即使想引导学生完成一些创新性探索实验, 也很 难开展和进行相关尝试。很多实验只能开设最为基础的实验, 这些实验都是验证性实验, 缺 乏设计性、综合性实验内容。实验结果的评估仅是依靠教师评定为主, 依据学生表现、遵章 守纪、实验报告撰写规范与否、数据处理得当与否、实验结论正确与否等作为主要评价依据, 完全忽视了学生在实验中的主体作用和他们在实验中的思考与探索。在工程教育和“卓越工程 师培养计划”的大背景下, 原有的教学体系已严重影响了人才培养质量, 不能满足工程教育“以 学生为中心”的培养目标和毕业要求, 亟需改革课程内容和教学方案、评价考核方式, 适应新 的培养方案。

\section{2. 教学内容的设置}

创新人才既要有深厚的理论基础, 又要较强的实践能力和创新能力。为了我们在修订新 的培养方案时, 对《无机材料综合实验》大纲进行相应修订。本课程目的是使学生通过材料 综合设计和试验研究, 使学生理解和掌握无机材料理论知识、专业知识和实践技能, 掌握正 确设计与研究思路、步骤和方法, 了解和掌握材料检测用标准方法, 了解和掌握配方计算与 实验数据获取与处理、实验结果分析与讨论的方法和手段, 掌握综合设计与研究报告的编写 方法。进一步巩固、深化和应用无机材料知识和技能, 培养实事求是的科学态度、严谨的工 作作风、坚忍不拔的意志, 培养和提高学生的基本技能、科学研究和工程设计能力、实验操 作能力、创新能力以及运用基本理论和方法分析和解决实际问题的能力, 为学生的毕业设计

（论文）、就业与创业奠定基础。《无机材料综合实验》成为我院无机专业比较重要的一门 综合实践类课程。

根据教学目的, 《无机材料综合实验》确定以水泥、玻璃、陶瓷的制备过程为主要内容。 学生在整个实验过程中, 分组设计一个无机非金属材料, 包括原料的选择-配方计算-工艺方案 设计-制备-相关性能测试及结构分析等全过程训练, 培养学生解决无机材料工程及科学研究问 题的能力与素质。学生能够根据实验所得现象进行分析, 并通过查阅文献, 解释实验现象。 在整个实验过程中学生进行分工合作, 相互配合有效完成相关任务。指导老师会根据科研发 展和材料进步不断更新制备材料的种类, 表 1 列出2015-2017年课程设置的实验内容。

表1 2015-2017年课程的实验内容设置情况

\begin{tabular}{|c|c|c|}
\hline 2015年实验内容设置 & 2016年实验内容设置 & 2017年实验内容设置 \\
\hline 类羊脂玉乳浊玻璃制作 & 类羊脂玉乳浊玻璃制作 & 类羊脂玉乳浊玻璃制作 \\
青花瓷的仿制和性能测试 & 青花瓷的仿制和性能测试 & 开片瓷的制备 \\
五彩琉璃饰品制作 & 五彩琉璃饰品制作 & 五彩琉璃饰品制作 \\
汝窑瓷的仿制和性能测试 & 汝窑瓷的仿制和性能测试 & 西瓜瓷壸的仿制 \\
& 变色水泥 & 盖板玻璃的设计和制备 \\
\hline
\end{tabular}

通过学习, 学生能结合典型材料生产工艺流程特点, 根据材料的性能自行设计材料的组 成, 通过一系列工艺制备这种材料, 并通过实验手段检测材料的性能, 看是否达到预期的目 的, 同时开展面向工艺过程的材料环境负荷辨识分析, 使学生认识到材料四要素中“合成与加 工”内容与成分结构、性质与使用性质各要素间的关系, 明确合成加工过程将改变材料的组织、 成分与结构, 进而影响到材料的使用性能这一思路和方法。如此教学内容的设置, 激发学生 的实验兴趣和动手能力, 鼓励学生积极参与老师的科研活动, 提高学生的科研和创新能力。 特别是将传统材料的制备过程和艺术设计相互融合, 减少了课程的枯燥无趣, 大大提升学生 的兴趣。 


\section{3. 探索开放式的实验教学模式建立}

传统的实验教学方法是灌输式, 学生围着老师转, 少有学生能动介入, 忽视了学生个体 的特性。我们在构建无机材料工程型人才实践教学体系以来, 不断优化实践教学方式、教学 方案、评价模式, 探索开放式教学模式, 形成鲜明的教育、教学特色, 激发学生主动性。

对于创新性综合实验, 根据学生特性让他们进行适当组合, 动手能力强和理论基础扎实 的学生相结合, 形成3-4人的实验小组。将实验室进行开放, 实验小组可根据自己的时间来预 约实验, 根据所拟定实验方案的时间规划, 提出相关仪器设备、原材料、其它开展实验的必 要物质。在无机材料的性能设计方面可使用计算机仿真设计软件。鼓励学生自行策划、设计 和创造。不仅使学生对课本知识有了深入的理解, 而且使学生的思维能力、动手能力、设计 能力、团队合作能力得到了很大的提高, 实现了从理论水平的提高到工程能力提高的转变, 取得了较好的效果。

“无机材料综合实验”改革亮点：（1）让学生组队自行设计一个材料, 包括原料的选择配方计算-工艺设计-制备-性能测试-结构分析-环境影响分析等全过程训练; (2) 不固定上课 时间, 学生自行安排, 学期末提交作品和实验报告; (3) 对每一件作品编写一首诗歌或散文, 融入传统材料的历史, 让学生的理解更加生动。

开放式的实验教学环节, 层次分明, 可以因材施教, 优才优育, 既有利于培养学生独立 从事无机材料研究和开发的基本技能和工程实验能力, 又使部分优秀学生具有一定的创新和 研究能力, 为将来进一步深造和企业工作打下扎实的基础。

\section{4. 以学生为本的考核机制改革}

已有的成绩评定体系都存在一个弊端, 开放性实验课程开设后, 学生自行设计实验方案, 实验时间根据实验内容而定, 老师不能全程跟踪学生, 不能对学生平时操作进行打分, 学生 自主性增大，如何合理评价成绩?

我们建立以学生为本的多层次、综合性评价办法, 改变单一以实验操作或实验报告为主 的评价方式, 并且逐渐弱化实验报告在评价中的比重, 不以实验报告论英雄, 这种机制提高 学生上课的积极性, 引导学生综合素质的提高, 全面评价学生运用知识和技能的能力。除了 对学生动手能力的培养, 还增加对学生团队协作, 领导能力, 表达能力的培养。课程除了让 学生完成实验外, 还增加期末课程汇报内容, 要求学生按照分组进行ppt汇报, 增加专家打分 环节, 专家对汇报内容进行打分。在此基础上, 评价内容更加细化, 包括平时实验操作和表 现, 综合实验技术路线和方案, 综合实验效果, 实验报告, 实验体会与感想, 同小组成员互 评, 不同小组之间互评, 专家评定等, 实验成绩来自多方面的综合评价, 既包括教师和专家 的评价, 也有来自学生之间的评价, 避免了评价的片面性, 避免传统的评价注重认真和实验 报告撰写规范者得高分现象, 通过实验考评方法的变革可以体现出对学生的创新能力关注, 可以促进和激发创新型学生的表现。最终成绩组成为平时成绩, 实验报告评价, 其它成绩, 其中平时成绩由指导教师和组内人员互评分组成, 实验报告成绩由任课教师根据实验报告和 课程ppt汇报综合评定，其它成绩由组间根据课程ppt汇报成绩相互评定。

这种考核机制试行两个学期后, 学生和指导教师都对考核办法感到满意, 学生成绩满足 正态分布, 同一个组的学生由于贡献不同给出公平的分数, 学生的积极性提高。

\section{5. 结束语}

我们改革工程专业培养目的相适应的无机材料卓越工程师模块实验课的教学内容、教学 方式、评价模式和考核方式, 在课堂教学中引入创新设计理论和观念, 形成基于工程和创新 思维能力培养的无机材料综合实验教学体系, 构建了 “厚实基础、注重兴趣、强调综合、强 化实验、自主学习、提高能力、不断创新”的《无机材料综合实验》完整创新实践教学新模式。 


\section{References}

[1] Edward F. Crawley et al. Rethinking Engineering Education: The CDIO Approach. Higher education press, 2009.

[2] WANG Hong-bo, XIE Bing, ZHANG Hua, et al. Study on experimental course teaching reform of solid waste treatment and disposal. Journal of Shandong Jianzhu University, 27(2012)351-354.

[3] Tong Shaocheng, Yu Ziqiang, Li Xin. The exploration and practice of constructing the training system for students' practice and innovation ability in engineering colleges $[\mathrm{J}]$. China University Teaching, 3 (2012)28-30.

[4] GONG Zhi-qiang, CHEN Hai-bo, XING Gang. DISCUSSION ON TEACHING REFORMS OF "PRINCIPLES OF MACHINERY"BASE ON THE TRAINING PLAN FOR "EXCELLENT ENGINEERS". Journal of Chaohu College, 17 (2015)136-139.

[5] LIU Guo-Liang, GAO Hai-tao. Reforming Experimentation Teaching, Improving Students Practice and Innovation. Journal of Luoyang Institute of Science and Technology (Natural Science Edition), 9(2010)94-96. 